\title{
Esthetic Frenectomy Using Bilateral Pedicle Approach
}

\author{
Marie Louisa*, Trijani Suwandi \\ Departemen of Periodontic, Faculty of Dentistry, Universitas Trisakti, Jakarta, Indonesia \\ *Email: marielousiasunardy@gmail.com
}

\begin{abstract}
Fraenal attachments are thin folds of muscle that attach the lips to the alveolar mucosa, and aberrant fraena have been associated with gingival recession and diastema. These aberrant fraena are detected by applying tension to see the blanching produced due to ischemia in the region. A fraenectomy is a surgical procedure used to correct an aberrant fraenum. This case report highlights an alternative fraenectomy technique used to achieve primary midline closure and avoid scar formation. A 22-year old female was referred by her orthodontist to undergo an upper labial fraenectomy during orthodontic treatment. This patient was treated using Archer's classical fraenectomy technique with a bilateral pedicle graft. A bilateral pedicle graft may help to achieve primary closure, thus avoiding scar formation after a fraenectomy.
\end{abstract}

Keywords: aberrant fraena, fraenectomy, bilateral pedicle graft, aesthetics

\section{Introduction}

Labial fraenal attachments are thin folds of the mucous membrane with enclosed muscle fibres originating from the orbicularis oris muscle of the upper lip that attaches the lips to the alveolar mucosa and underlying periosteum. Depending on the extension of the attachment, fraena have been classified as mucosal, gingival, papillary and papilla penetrating (Fig 1). A mucosal attachment includes fraenal fibres attached up to the mucogingival junction, and a gingival attachment includes fraenal fibres inserted within the attached gingiva. A papillary attachment includes fraenal fibres extending into the interdental papilla. A papilla penetrating attachment includes fraenal fibres crossing the alveolar process and extending up to the palatine papilla. Clinically, papillary and papilla penetrating fraena are considered to be pathological and have been found to be associated with papilla loss, recession, diastema and difficulty brushing [1,2]. 


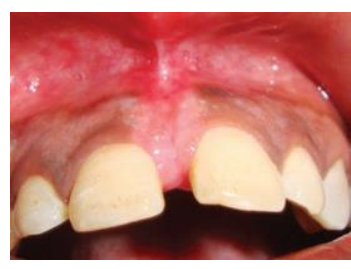

a.

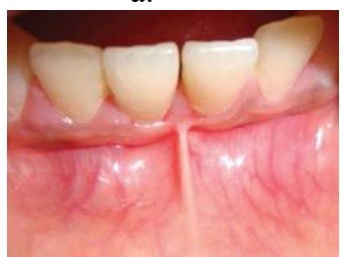

c.

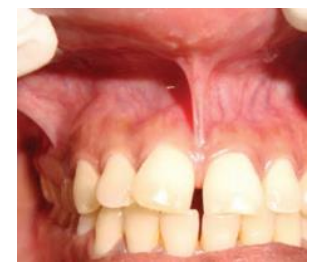

b.

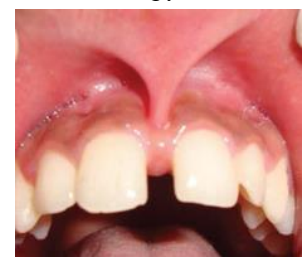

d.

Fig 1. (a) Mucosal fraenal attachment. (b) Gingival fraenal attachment. (c) Papillary fraenal attachment. (d) Papilla penetrating fraenal attachment [1,2]

Abnormal or aberrant fraena are detected visually by applying tension to see if there is movement of the papillary tip or blanching due to ischemia in the region (blanch test). A positive blanch test result indicates a narrow or no apparent zone of attached gingiva along the midline, so it is necessary to perform a fraenectomy for functional and aesthetic reasons [3]. There are numerous surgical techniques available for the removal of the labial fraenum. In the standard fraenectomy procedure by Archer and Kruger, the fraenum, interdental tissue and palatine papilla are completely excised, leading to exposure of the underlying alveolar bone, and thus, scarring. Although this technique results in an unaesthetic scar, it has been advocated to ensure the removal of the muscle fibres to prevent a midline diastema relapse [3,4].

Miller's technique was introduced in 1985 when Miller described a surgical technique combining a fraenectomy with a laterally positioned pedicle graft. If the fraena is enlarged, a gingivoplasty is performed to reduce it to an appropriate size. The closure across the midline by laterally positioning the gingiva and healing by primary intention result in attached gingiva across the midline. The interdental papilla remain undisturbed because no attempt was made to dissect the trans-septal fibres $[3,5]$. Since aesthetically and functionally better results have been obtained, Miller's technique was used in this case, and the result is presented in the following case report.

\section{Case and Management}

A 22-year old female was referred by her orthodontist to undergo an upper labial fraenectomy during orthodontic treatment. This patient's medical history was noncontributory, and her clinical examination revealed a papillary penetrating type of fraenal attachment. The blanch test was positive (Fig 2). A full complement of teeth 
was present with adequate buccal vestibular depth. This patient's oral hygiene was good, and there were no clinical signs of gingival inflammation.

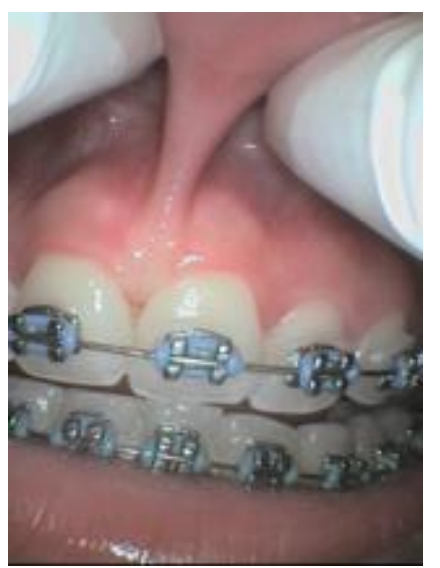

a.

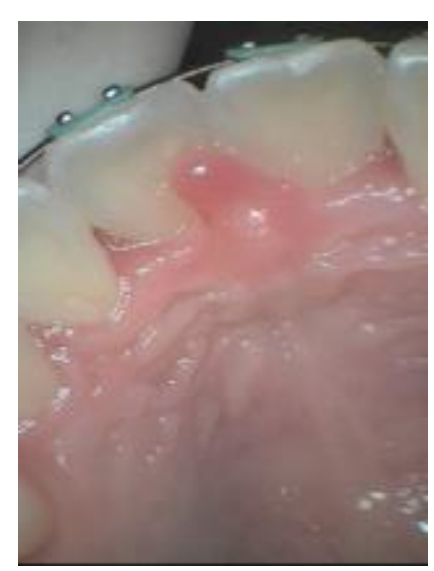

b.

Fig 2. (a) Buccal aspect and (b) palatal aspect of the fraenal attachment.

The maxillary anterior region was anaesthetized via local infiltration on the buccal and palatal aspects. Then, a horizontal incision using a no. 15 Bard-Parker knife was used to separate the fraenum from the base of the interdental papilla. This incision was extended apically up to the vestibular depth to entirely separate the fraenum from the alveolar mucosa. Any fraenal tissue remnants in the midline or on the under surface of the lip were excised.

Two oblique partial-thickness incisions were then placed in the adjacent attached gingiva, beginning 2-3 $\mathrm{mm}$ apical to the marginal gingiva, up to the vestibular depth. In order to mobilize the flap, the gingival and alveolar mucosa between these two incisions was undermined by sharp dissection. The flaps were mobilized mesially and sutured to each other on the medial side and laterally to the adjacent intact periosteum of the donor site using 4-0 silk suture, completely covering the underlying defect created by the initial fraenal excision (Fig 3a, b).

There was no attempt made to dissect the trans-septal fibres between the approximating central incisors. A gingivoplasty of the excess palatal tissue in the interdental area was performed, preserving the integrity of the interdental papilla (Figure 3c). Postoperative instructions were provided, and analgesics and antiseptic mouthwash were prescribed for five days during the post operative period. The sutures were removed on the 10th day (Figure 4) and the patient was scheduled for follow-up visits (Fig 5-7). 


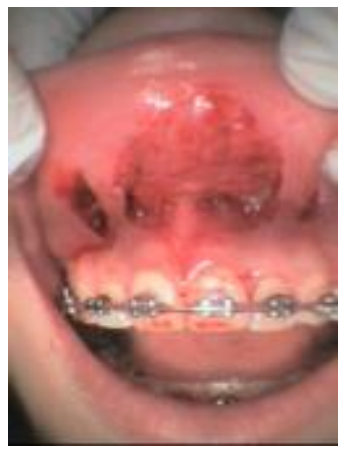

a.

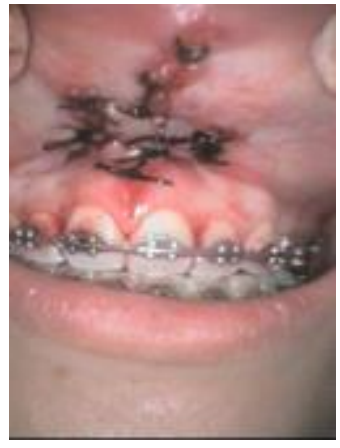

b.

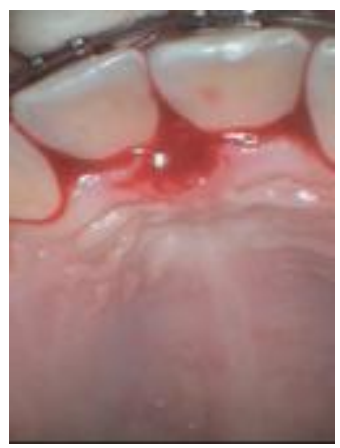

c.

Fig 3. (a) After the fraenal tissue was excised, two oblique parallel partial thickness incisions were made. (b) After the pedicles were mobilized mesially, both were sutured, completely covering the underlying defect. (c) Gingivoplasty of the excess palatal tissue in the interdental area.

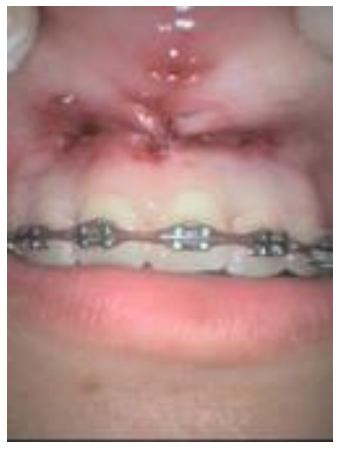

a.

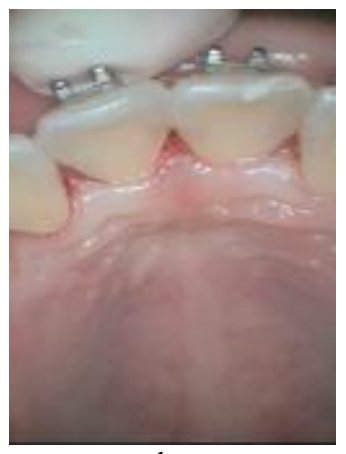

b.

Fig 4. (a) Suture removal on the 10th day. (b) Palatal aspect.

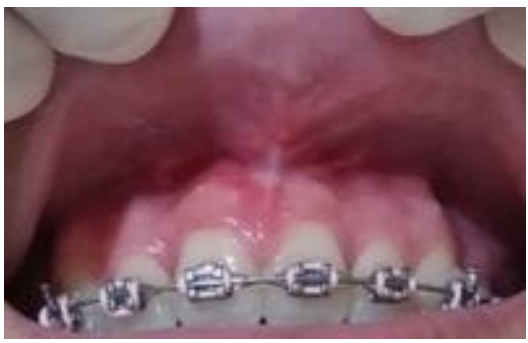

a.

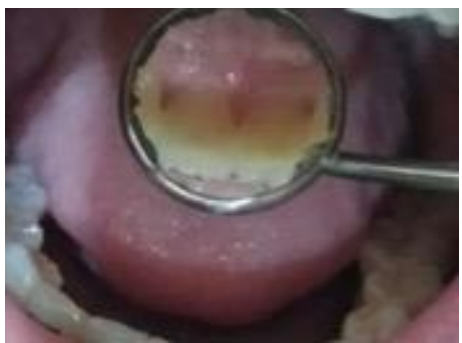

b.

Fig 5. Recall visit 1 month after surgery. (a) Buccal aspect and (b) palatal aspect. 


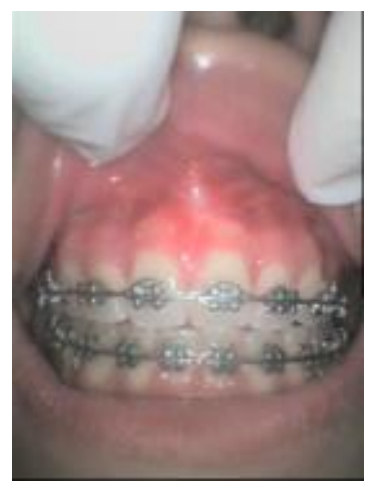

a.

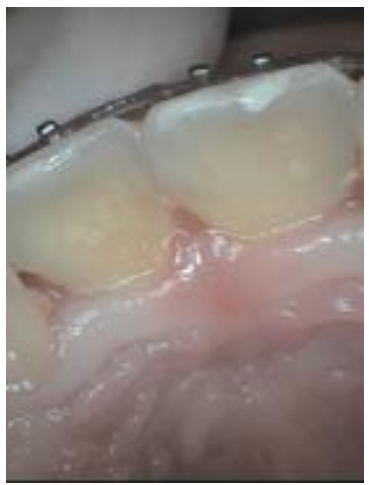

b.

Fig 6. Recall visit 2 months after surgery. (a) Buccal aspect and (b) palatal aspect.

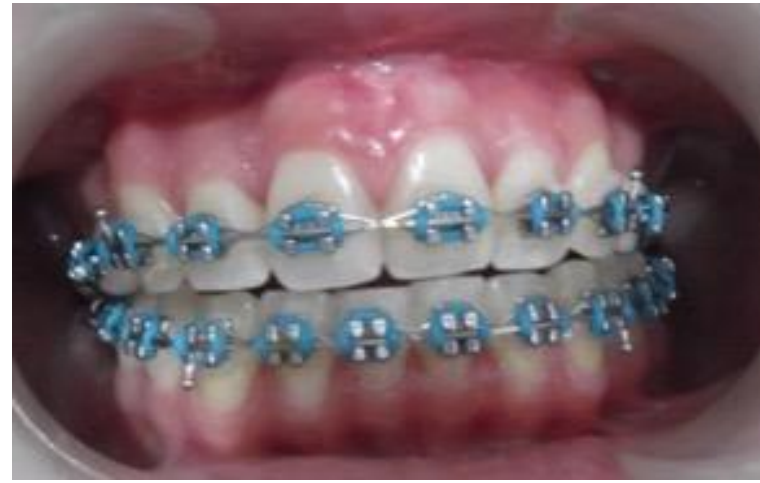

Fig 7. Recall visit three months after surgery.

\section{Discussion}

In the era of periodontal plastic surgery, more conventional and precise techniques are being adopted to create more functional and aesthetic results. The methods available include Archer's classical fraenectomy technique, a Z-plasty incision, a fraenectomy with a soft-tissue graft and Miller's technique [4,6] Archer's classical technique (often called a V-shaped or diamond incision) is performed with two parallel incisions on each side of the fraenum joined in the vestibule by a scissor cut. The wound edges are closed with a single suture. This technique has been reported to leave a contracture scar that can lead to periodontal problems, as well as the loss of the interdental papilla between the central maxillary incisors $[3,4]$.

In the Z-plasty incision, a vertical incision is made along the fraenum from the gingival margin to the vestibule. Then, two incisions are made at each end of the primary incision at angles of 60 degrees, pointing in opposite directions, forming a Z-shaped incision. The two mucosal flaps, without the periosteum, are elevated and 
sutured in reverse positions (Fig 8). This technique permits better distribution of the scar contracture lines but is more complicated and more surgically demanding [7].

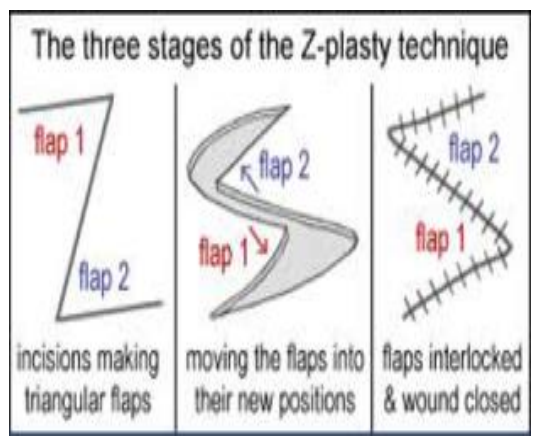

a.

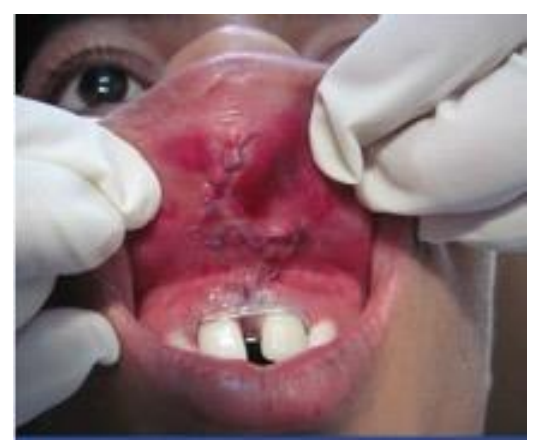

b.

Fig 8. (a) The three Z-plasty stages. (b) Clinical view. ${ }^{7}$

Coleton and Lawrence used a free gingival graft combined with a fraenectomy in order to avoid creating a scar and to cover the wound area completely. However, the mismatched gingival colour in the midline and the need for a second surgical site to collect donor tissue complicate this technique [3,5].

Miller presented a surgical technique combining a fraenectomy with a laterally positioned pedicle graft (Fig 9). The closure across the midline via the laterally positioned gingiva and healing by primary intention resulted in aesthetically acceptable attached gingiva across the midline. No attempt was made to dissect the transseptal fibres; therefore, the interdental papilla remained undisturbed. Furthermore, there was no need for a second surgical donor site, thus reducing the patient's morbidity [3].
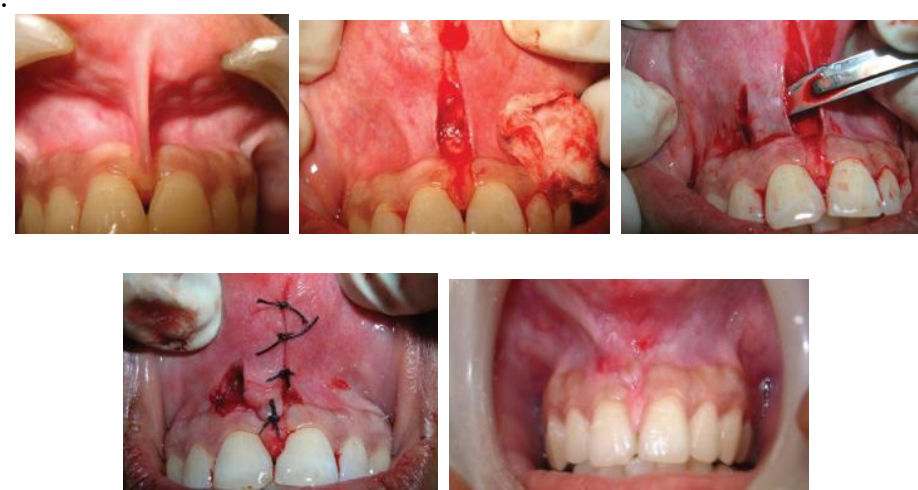

Fig 9. Fraenectomy with laterally positioned pedicle graft (Miller's technique) [3]

If the wound produced after a fraenectomy is large due to a thick, broad or hypertrophic fraenum, a lateral pedicle graft might not provide complete wound coverage. Therefore, a modification was made by using bilateral pedicles (Fig 10). 
These pedicles maintain the width of the attached gingiva without compromising the colour match because of the resulting scar formation. Moreover, covering the $\mathrm{V}$-shaped defect with the pedicles not only helps to attain healing by primary intention, it also avoids the formation of an unaesthetic scar. The patient's discomfort is also minimized when compared to conventional fraenectomy procedures in which the defects are left substantially open. In addition, it preserves and enhances the attached gingiva at the site previously occupied by the labial fraenum, helping to maintain the periodontal health of the involved teeth postoperatively $[8,9]$.

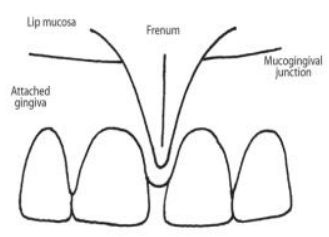

a.

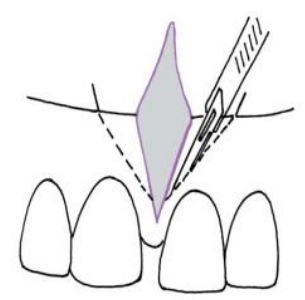

d.

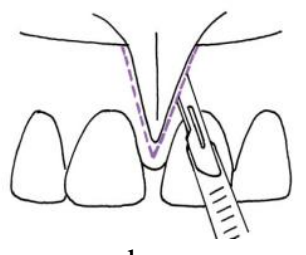

b.

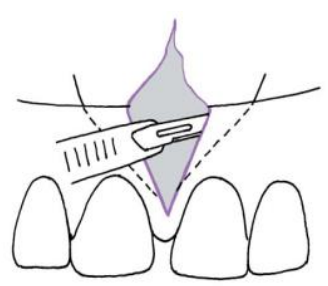

e.

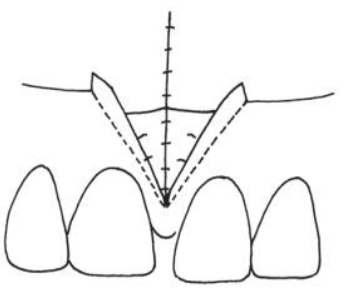

g.

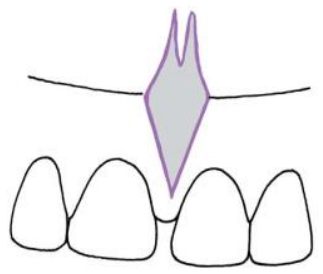

c.

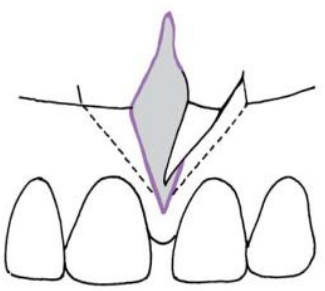

f.

Fig 10. (a) Thick hypertrophied fraenum. (b) V-shaped external bevel incision. (c) V-shaped defect after the fraenal excision. (d) Oblique partial-thickness incision on the adjacent attached gingiva extending beyond the mucogingival junction. (e) Partial-thickness dissection of the attached gingiva. (f) Triangular pedicle of the attached gingiva. ( $\mathrm{g}$ ) Bilateral triangular pedicle sutured at the centre covering the underlying defect. [5]

In this case, although the diastema was absent, the muscle pull of the thick labial fraenum could cause a relapse of the orthodontic treatment. As a preventive tactic to avoid relapse and scar formation, a bilateral pedicle approach fraenectomy was carried out. As a result, the newly formed attached gingiva along the midline, which 
contains collagenous fibres, may have a bracing effect and prevent the reopening of the diastema $[6,8]$.

\section{Conclusion}

The bilateral pedicle approach fraenectomy performed in this case report has certain distinct advantages when compared to the other approaches. For example, the healing takes place via primary intention, thus avoiding the formation of an unaesthetic scar. In addition, the attached gingiva matches the adjacent tissue in the midline, which is pleasing to the individual. Moreover, there is no recession of the interdental papilla because the trans-septal fibres are not severed. Finally, the attached gingiva in the midline may have a bracing effect, which helps to prevent orthodontic relapse.

\section{References}

1. Priyanka M, Sruthi R, Ramakrishnan T, Emmadi P, Ambalavanan N. An overview of frenal attachments. J Indian Soc Periodontol. 2013;17(1):12.

2. Díaz-Pizán ME, Lagravère MO, Villena R. Midline diastema and frenum morphology in the primary dentition. Journal of dentistry for children. 2006 Jan 1;73(1):11-4.

3. Chaubey KK, Arora VK, Thakur R, Narula IS. Perio-esthetic surgery: Using LPF with frenectomy for prevention of scar. J Indian Soc Periodontol. 2011;15(3):265.

4. Delli K, Livas C, Sculean A, Katsaros C, Bornstein MM. Facts and myths regarding the maxillary midline frenum and its treatment: a systematic review of the literature. Quintessence Int. 2013;44(2).

5. Bagga S, Bhat GS, Thomas BS. Esthetic management of the upper labial frenum: a novel frenectomy technique. Quintessence Int.. 2006;37(10).

6. Indurkar M, Maurya A. Ortho-Perio interdisciplinary approach for management of midline diastema with high frenum attachment: A Case Report. Clin Dent. 2017;11(1).

7. Agarwal A, Kapahi R. Labial frenectomy through Z plasty. J Clin Diagn Res. 2012; 6:12.

8. Desai AJ, Bedi S, Gowda TM, Thomas R, Mehta DS. Bilateral pedicle approach for esthetic management of upper labial frenum. J Interdisciplinary Dent. 2015;5(1):27.

9. Hupp JR. Wound repair. In: Peterson LJ, Ellis E, Hupp JR, Tucker MR (eds). Contemporary Oral Maxillofac Surg. 2004:53-55. 